\title{
Research on Power System Fault Diagnosis Based on Bayesian Networks
}

\author{
Guofeng Yang \\ School of Economics and Management \\ North China Electric Power University \\ Beijing, China \\ Ningxia Electric Power Company \\ Yinchuan, China \\ yangguofeng@nx.sgcc.com.cn \\ Qingming Xiao \\ Ningxia Electric Power Company \\ Yinchuan, China \\ xiaoqingming@nx.sgcc.com.cn
}

\author{
Hong Ouyang, Jiakui Zhao \\ Beijing China-Power Information Technology Co., Ltd. \\ State Grid Electric Power Research Institute \\ Beijing, China \\ \{ouyanghong, zhaojiakui\}@sgepri.sgcc.com.cn
}

Tingshun Li, Jing Zhou

School of Control and Computer Engineering

North China Electric Power University

Beijing, China

\{ashur0925, zhoujing108\}@163.com

\begin{abstract}
Aiming at the incompleteness and uncertainty of information existing in power system fault diagnosis, a new fault diagnosis approach based on Bayesian network is proposed in this paper. Through the Bayesian network of structure learning and parameter learning, a power system fault diagnosis model based on Bayesian network has been proposed. Conditional probability table describes the connection degree between various factors in quantity. Diagnostic results of instance proved the effectiveness and superiority of the proposed method.
\end{abstract}

Keywords-power system; fault diagnosis; Bayesian network; structure learning; parameter learning.

\section{INTRODUCTION}

With the rapid development of scientific technology, the scale and structure of power system continue to expand and become complicated. In the process of power system operation, natural or man-made interference often occurs and the failure is difficult to avoid. Therefore, adopting effective method to diagnosis the fault of power system accurately, finding out the fault components, reducing manpower, material resources and economic losses, appear particularly important. Now, with the further development of artificial intelligence, especially machine learning, data mining, etc, many theories and methods are offered to diagnosis the fault. Such as expert system, artificial neural networks, optimization method, fuzzy sets theory etc. Although these theoretical researches have scored some achievements, there are still certain limitations.

The uncertainty of power system operation, the diversity, complexity and associated level-oriented of gathering information, cause detection randomness and uncertainty. Combining graph model theory and statistical knowledge, Bayesian network provides a method to describe causal relationship between variables. Using probability theory to handle the uncertainty between different knowledge for conditions related, so it thus become one of the models in the field of uncertain knowledge representation and reasoning..
Applying the Bayesian network to power system fault diagnosis, can solve incomplete and uncertainty. Using the Bayesian network structure learning algorithm to obtain a precise power system fault diagnosis model in qualitative, using the Bayesian network parameters learning algorithm to obtain the table of conditional probability and reflect the link degree between components in quantitative. Through reasoning algorithm further achieve the power system fault diagnosis under the uncertainty and incomplete information.

\section{THE SUMMARY OF BAYESIAN NETWORKS}

\section{A. Description of bayesian network}

Bayesian network[1] also called reliability network, is the expand of Bayes method. A Bayesian network is a directed acyclic graph(DAG), which is organized by node representing variables and directed edges connecting these nodes. Nodes represent random variables, the edges among nodes represent the relationship between nodes (the father node pointing its offspring node). The conditional probability express relationships strength. Without father nodes, prior probability can express information. Nodes variable can be the abstract of any problem, such as test value, observations, etc. It is applicable to express and analysis of uncertainty and probabilistic events, applied to a conditional relies on multiple controlled factor of decision making, can make reason among incomplete, inaccurate or uncertain knowledge or information.

A Bayesian network with a group of variables $V=\left\{X_{1}, X_{2}, \cdots, X_{n}\right\}$ contains the following two parts:

(1)A network structure $S$ express conditional independencies relationship among variables set $V$.

(2) A table of conditional probability associated with each variable.

The network structure $S$ is a directed acyclic graph, the nodes in $S$ is one-to-one mapping to variables 
$X_{1}, X_{2}, \cdots, X_{n}$ in $V$. Nodes without arc connected

represent they are conditionally independent. $S$ and

$P$ define the joint probability distribution of $V$. According to the nature of conditional independencies, joint probability distribution is

$$
P(V)=P\left(X_{1}, X_{2}, \cdots, X_{n}\right)=\prod_{i=1}^{n} P\left(X_{i} \mid P a\left(X_{i}\right)\right)
$$

For each variable $X_{i}, \operatorname{Pa}\left(X_{i}\right)$ is the father set of $X_{i}$.

If the event $X_{i}$ is constituted by several small events $\left\{X_{i}\right\}$ $(\mathrm{i}=1 \ldots \mathrm{m})$, all related variables observation results are known except $X_{i}$, according to the Bayesian theorem, conditional probability is:

$$
P\left(x_{i} \mid V\right)=\frac{P\left(x_{i}, V\right)}{P(V)}=\frac{P\left(x_{i}, V\right)}{\sum_{i=1}^{m} P\left(x_{i}, V\right)}
$$

\section{B. Bayesian network learning and reasoning}

Learning the Bayesian network is to seek a network includes searching for a directed acyclic graph(DAG) structure and gain the table of conditional probability related to all nodes in DAG. The former called network structure study, the latter is called network parameters learning. Through the network structure and data sets can determine parameters, so structure learning is the core of learning Bayesian network. Effective structure learning methods and algorithm is the foundation of constructing optimal network structure. A Bayesian network can reflect qualitative information between variables, also can reflect the quantitative information.

Qualitative information is reflected by arc, representing the relationships among variables; Quantitative information is reflected by relationship strength among variables, namely represented by the table of conditional probability between node and his father node.

Uncertainty reasoning methods mostly developed on the basis of uncertainty reasoning method, or rather to expand or improve certainty reasoning. The most common way is to put instructions degree of certainty data attached to reasoning rules, and thus developed subjective Bayes method, the deterministic method and the D-S evidence theory etc. Bayesian network reasoning actually know results obtained parameters process, fault diagnosis reasoning is to obtain the reason of fault by using the fault information, says node events and the state of the fault occurred in event joint probability is the largest. According to the diagnosis reasoning directions, Bayesian have three important reasoning methods: reason pushed to the results, the Bayesian network arbitrary connected the two nodes represent direct cause-effect; Results pushed to reason, according to the known fault and node probability information, deduce trigger fault reasons; Explain reasoning, need to infer all the possible reasons that may lead to failure.

\section{Tectonic Bayesian Network Diagnosis Model}

\section{A. Power system protection action principle}

Electric power system fault diagnosis includes: line, transformer and bus. Relay protection of lines and transformer protection is 3-sectional protection: main protection(m), only protect circuit itself, the scope of protection is generally $75 \%$ of the full-length; The first backup protection(p), protecting the whole length of circuits, when the main protection didn't start, first backup protection start automatically; The second backup protection(s), the adjacent elements fault but the protection not start, the second backup protection start automatically to removal fault. Bus relay protection is only main protection, when the bus is fault, relay protection start automatically.

\section{B. Tectonic bayesian network model}

According to the literature[2] the local power relay protection system as shown in figure 1, structure the Bayesian network model of components $B_{1}$.

$\mathrm{CB}, \mathrm{A}$ and L separately represent circuit breaker, bus and circuit. L, R in lines separately represent the left and right end of export protection. The values of the relay protection node $s_{j} 0$ or 1 separately represent in "don't start" or "start" state. The values of components section $C_{i} 0$ or 1 separately represent in "normal" or "fault" state.

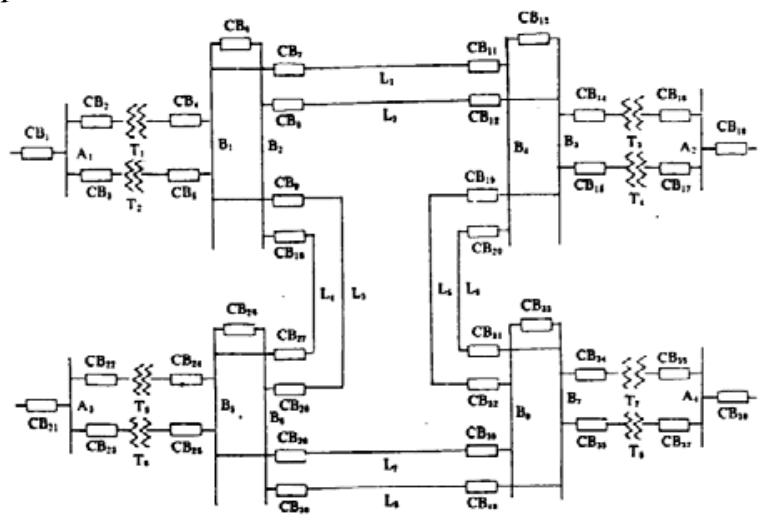

Figure 1. local power system protection figure

According to the relay protection action theory of component $B_{1}$, construct the fault diagnosis Bayesian network diagram of component $B_{1}$, as figure 2 shows.

Identify applicable sponsor/s here. (sponsors) 


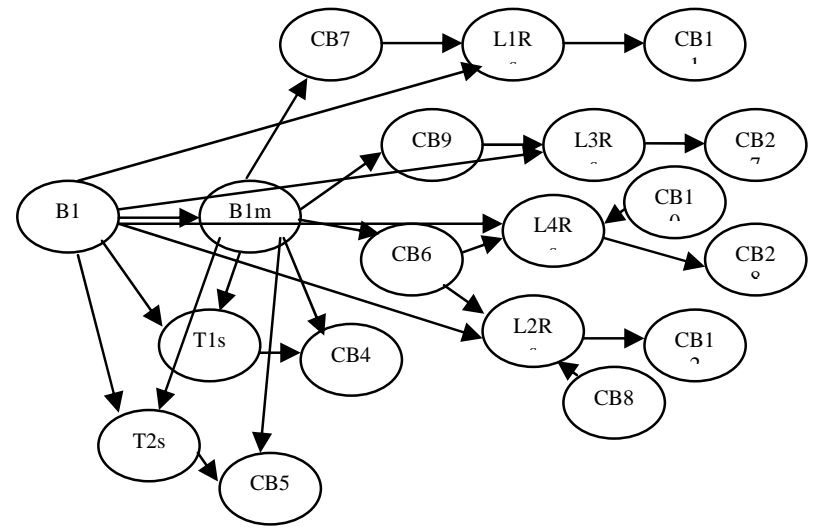

Figure 2. the Bayesian network of component $B_{1}$

\section{Bayesian network structure learning}

Analysis the Bayesian network diagram of component $B_{1} \quad$ the direct access $B_{1} \rightarrow B_{1 m} \rightarrow C B_{6} \rightarrow L_{2} R_{s} \rightarrow C B_{12}$ represent: $B_{1}$ is in fault, $B_{1 m}$ act and disconnect $C B_{6}$, if $B_{1}$ is in fault and $C B_{6}$ don't disconnect, $L_{2} R_{s}$ act and disconnect $C B_{12}$. The fault diagnosis model established with Bayesian network can clearly intuitively express the relationship among components, all levels of protection and the breakers.

\section{Bayesian network parameters learning}

Computing the prior probability of element node fault, the equipment continue operate a period of time $t$, the probability of failure happen is the probability of fault time intervals for $\mathrm{t}$.

$$
P\{T \leq t\}=1-e^{-w t}
$$

$\mathrm{T}$ is the equipment continuous trouble-free operation time, with literature [3] the data takes $\mathrm{w}$, take $\mathrm{t}=0.5$, computing the prior probability of element node fault.

TABLE I. THE PRIOR PROBABILITY TABLE OF ELEMENT NODE FAULT

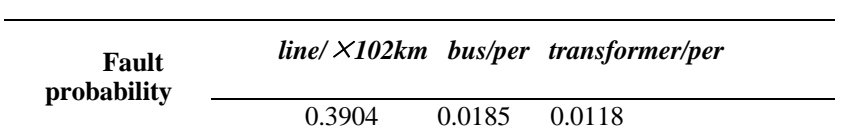

Calculate the probability of node protection device don't start, use refusing action times divided by requirements times. The misoperation probability of relay protection node, first calculate relay protection in fixed time of misoperation frequency, then follow the formula 3 to calculate misoperation probability. The relay protection refusing action, misoperation probability as shown in table 2[4]. FP means failure probability, LMP means line main protection, BMP means bus main protection, TMP means transformer main protection, $\mathrm{B}$ means breaker.
TABLE II. RELAY PROTECTION REFUSE ACTION, MISOPERATION PROBABILITY TABLE

\begin{tabular}{ccccc}
\hline FP/\% & LMP/per & BMP /per & TMP/per & B/per \\
\hline misoperation & 0.0085 & 0.0022 & 0.0039 & 0.0268 \\
\hline Refuse action & 0.0019 & 0.0013 & 0.0027 & 0.0145
\end{tabular}

On the basis of the above analysis data and protect action theory, it can be sure the conditional probability of all nodes. Using the similar characteristics of the relay protection node conditional probability, first determine several key nodes conditional probability of relay protection , and then the generation gonging into all the node and obtain the conditional probability. Among them, 0 means components normal, -1 means components failure; In the relay protection, 0 means protecting state startup, 1 means protecting state didn't start. CP means conditional probability. The conditional probability of L1Rs as shown in table 3 [5].

TABLE III. THE CONDITIONAL PROBABILITY TABLE OF L1RS

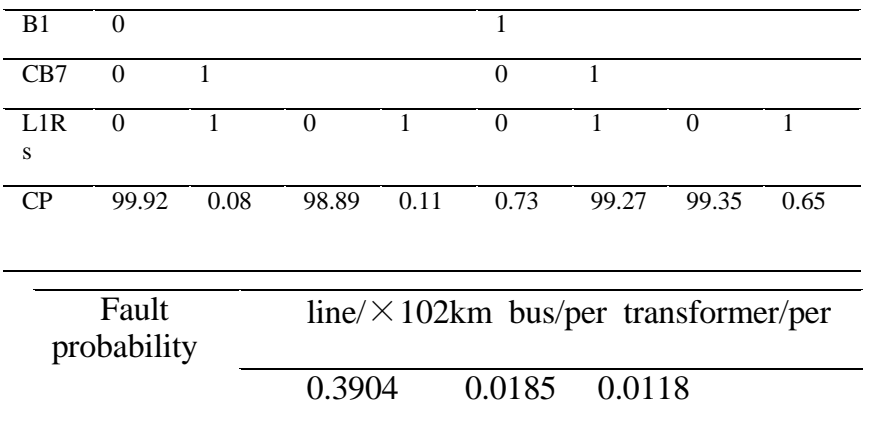

The data in L1Rs conditional probability shows on the condition of B1 normal and relay CB7 startup, the probability of L1Rs startup is $99.92 \%$, L1Rs don't startup is $0.08 \%$. On the condition of B1 normal and CB7 don't startup , the probability of L1Rs startup is 98.89\%, L1Rs don't startup is $0.11 \%$; On the condition of B1 is fault and relay CB7 startup, the probability of L1Rs startup is $0.73 \%$, L1Rs don't startup is 99.27\%; On the condition of B1 is fault and relay CB7 don't startup, the probability of L1Rs startup is $99.35 \%$, L1Rs don't startup is $0.65 \%$. Bayesian network reasoning is based on network model and the known information network nodes, using conditional probability calculation method in Bayesian theorem, to obtain the conditional distribution probability of decision-making node subsets, then make decision analysis with the results.

\section{SUMMARY}

In recent years, Bayesian network has a wide range of application in data mining, knowledge engineering and information retrieval. With the further development of artificial intelligence, especially machine learning and data mining, provide a wider space for the development and application of Bayesian theory. Bayesian network is one of important methods to deal with uncertain problems. It ha a solid theoretical foundation of mathematics, combining the prior information and domain knowledge, can easily handle incompletely data and mature approximation method. As for traditional fault diagnosis method, if the information is 
incomplete, the model will deviate and may draw a wrong diagnosis. Bayesian network method reflect the probabilistic relationship of all the correlative nodes. Through the change Bayesian network topology and reassess the conditional probability of missing information nodes, establish fault diagnosis Bayesian network model with the incomplete information. Bayesian network fault diagnosis method integrated a priori information and posterior information, can effectively avoid the subjective bias only with priori information and blind searching and calculation lacking sample information, and the noise influence only with a posteriori information.

\section{ACKNOWLEDGMENT}

This work was supported by the Science and Technology Project of Ningxia Electric Power Company entitled "Research and Application on the Formal Description of the Knowledge in Smart Grid", the Science and Technology Project of SGEPRI (State Grid Electric Power Research Institute) entitled "Research on the Information Processing and Typical Applications for Electric Internet of Things”, and the Science and Technology Projects of State Grid Corporation of China entitled "Research on the Integrated Supporting Technologies for Intelligent Marketing Business based on the International IEC-CIM/CIS Standard" and "Research on the Technologies of Analysis and Pre-Warning for Company Operation”.

\section{REFERENCES}

[1] F.V. Jason. Bayesian Networks and Decision Graphs. Springer Verlag, New York, 2001:18-25

[2] Wen Fushun, Han Zhenxiang. Fault Section Estimation in Power Systems Using Genetic Algorithm and Simulated Annealing. Proceedings of the CSEE, Vol.14 No.3, pp. 29-35, May,1994.

[3] Zhong Yulan, Wang Junyong, Shu Zhihuai. Statics of operation situation of protective relaying and automation devices of power systems in china in 2002.Power System technology,2003,pp:55-60.

[4] Wu Xin, Guo Chuangxin. Power system fault diagnosis approach based on Bayesian network. Proceedings of the CSU-EPSA, vol.17 No.4,pp:11-15,Aug.2005.

[5] Dong Chen. Applications and Research of the Bayesian Network in power system fault diagnosis. Shenyang University of Technology Press.2009.

[6] Guan Huiling, Han Jie. Equipment fault diagnosis expert system theory and practice. Electronic Industry Press. 2000(in Chinese). 